DOI: 10.4274/ejgg.galenos.2021.2-1

Eur J Geriatr Gerontol 2021;3(1):1-3

\title{
Using Ultrasound in the Assessment of Muscle
}

\author{
(1) Stany Perkisas ${ }^{1}$, (1) Kristoffer Brockhattingen², (1) Carly Welch ${ }^{3}$, (1) Gülistan Bahat ${ }^{4}$ \\ 1 University Centre for Geriatrics, ZNA (Ziekenhuis Netwerk Antwerpen), Antwerpen, Belgium \\ 2Odense University Hospital, Clinic of Geriatric Medicine, Svendborg, Denmark \\ 3 University of Birmingham, Medical Research Council-Versus Arthritis Centre for Musculoskeletal Ageing Research, Birmingham, United Kingdom \\ 4 istanbul University Istanbul Faculty of Medicine, Department of Geriatrics Medicine, Division of Geriatrics, Istanbul, Turkey
}

Of all organs, muscle is possibly the most underrated. First, it is seldom thought of as an organ. Second, for many years it was placed low on the ladder of interest around the medical community due to its apparent singular locomotor effects. Third, even if the only perceived function of movement seemed to fail, other causes were quickly to be blamed, most often neurological. Fortunately, during the past 30 years, the intrinsic worth of the muscle has been appreciated more. Slowly, it has become evident that muscle as an organ undertakes an important endocrine function, in addition to its vital role as the sole source of protein reserve in the body-truly indispensable for whole body metabolism (1). The recent insights to this "newly discovered" organ have enhanced interest in pathologic alterations, sarcopenia being one of the most important.

This chronic and, in old age, seemingly inevitable muscle affliction, is currently defined by the European Working Group on Sarcopenia in Older Persons (EWGSOP) as a progressive and generalised skeletal muscle disorder. It consists of deficits in three main components, being muscle quantity or quality, muscle strength, and physical performance $(2,3)$. The importance of sarcopenia is reflected in its relation with many negative health outcomes, of which perhaps the most evident being mortality. With an odds ratio (OR) of 3.6, it could be regarded as even more relevant than other comorbidities in older persons, such as heart failure (OR 1.66), dementia (OR 2.01) or even cancer (OR 3.02) $(4,5)$. Another factor is the issue of quality of life, which declines in patients with sarcopenia (3). Although sarcopenia has recieved its own ICD-10 code (6), there is still a lot of debate about the exact value of the different components described. Over the last years, physical performance has been viewed as either diagnostic criterion, severity grading assessment or as an outcome (7). Muscle strength seems to be better at predicting adverse outcomes (3), but this may be due to its ease of measurement in comparison to either muscle function or quality/quantity. The most difficult issue seems to be the most basic one, which is defining muscle mass. In primary sarcopenia, there are changes in both muscle quantity and quality, the latter being described as micro- and macroscopic aspects of muscle architecture and composition $(3,8)$. Despite these changes being clearly paramount in the genesis of sarcopenia, technological limitation so far have limited the transposition from knowledge to practice. In the current FACS-algorithm (Find cases-assessconfirm-severity) proposed by the EWGSOP2 for the diagnosis of sarcopenia, a proposition is made to measure either muscle quantity or quality. Unfortunately the algorithm does not specify what parameters of quality should be measured (3). This renders muscle mass a non-defining parameter, creating another obstacle in making an accurate diagnosis (7).

The key to overcome this problem is twofold. First, relevant anatomic and architectural changes should be defined. Second, the technique to measure these parameters should be widely available in clinical practice. Currently, both issues are still obstacles. The currently proposed techniques for muscle mass assessment are bioelectrical-impedancemetry, dual energy $X$-ray absorptiometry, computed tomography or magnetic resonance imaging (3). However, where the two former are incapable of looking into muscle quality parameters, the two latter are impossible to be used bedside. Therefore, we must turn to new technologies in order to advance.

To be clear, ultrasound is not a new technique. It has been present since the late 1950's and has continued to improve in ease of use and portability. Although its use in medicine is

Address for Correspondence: Stany Perkisas, University Centre for Geriatrics, ZNA (Ziekenhuis Netwerk Antwerpen), Antwerpen, Belgium Phone: +32-0-3-280-35-39 E-mail: stany.perkisas@zna.be ORCID: orcid.org/0000-0002-4192-327X

Received: 11.02.2021 Accepted: 12.02 .2021

Cite this article as: Perkisas S, Brockhattingen K, Welch C, Bahat G. Using Ultrasound in the Assessment of Muscle. Eur J Geriatr Gerontol 2021;3(1):1-3

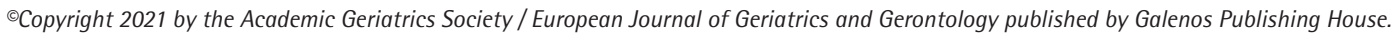


widespread, its application in musculoskeletal research is often limited. However, in recent years ultrasound was hypothesized as the perfect alternative for the current muscle assessment problems (9). As a portable, cheap and easy technique that can be performed bedside, ultrasound is positioned as a patientcentred diagnostic tool. In addition, ultrasound enables the physician to visualise a wide range of components of muscle arcitecture, hence underlining all the advantages of becoming the new standard tool in screening for the presence of sarcopenia. However, some obstacles still stand in the way of it being used as a first line tool.

Knowledge of the relevance of the different muscle components is still in its early phase. Although muscle thickness, crosssectional area, pennation angle, fascicle length and echointensity have been proposed earlier (9), other measurements such as elastography (10) or vascularisation could offer important information. Another issue is the lack of standardisation of measurements, which is a must in order to be able to compare research data. Until very recently this was only provided for a limited amount of muscles (9). Nowadays, standardisation for 39 muscles/muscle groups are present (11), making it possible for researchers worldwide to investigate effects of specific muscles. Until now, the quadriceps is the muscle most investigated as it is easy to measure and can be linked directly to measures of physical performance $(12,13)$. The final horde that it needs to take now, is the collection of reference data in different age cohorts and populations (13). This way, pathological values can be distilled, cut-off lines can be drawn and correlations can be made with the other aspects of sarcopenia-strength and function.

Hereby we call upon all researchers interested to look into this new and very exciting field of using ultrasound in muscle assessment. It is our firm belief that only through using ultrasound, the true diagnostical approach of muscle mass loss will be taken bedside -into clinical practice- where it so urgently is needed. This advancement needs worldwide collaboration, of which the first steps are already taken through the European initiative of SARCUS, a project of the European Geriatric Medicine Society (14). This project tries to answer the remaining gaps in knowledge that currently restrain the use of ultrasound in clinical practice. Besideds standardisation, current projects are acquiring reference data and looking into the most relevant muscle parameters to be linked with clinical outcomes. This way, the future hope is to include muscle ultrasound as a part of comprehensive geriatric assessment, giving a deeper understanding of how to better treat our older patients.

Keywords: Aging, clinical geriatrics, frailty, geriatric care management, sarcopenia, ultrasound

\section{Ethics}

Peer-review: Internally peer-reviewed.

\section{Authorship Contributions}

Concept: G.B., S.P., Desing: S.P., Literature Search: S.P., K.B., C.W., G.B., Writing: S.P., K.B., C.W., G.B.

Conflict of Interest: No conflict of interest was declared by the authors.

Financial Disclosure: The authors declared that this study received no financial support.

\section{References}

1. Argilés JM, Campos N, Lopez-Pedrosa JM, Rueda R, Rodriguez-Mañas L. Skeletal Muscle Regulates Metabolism via Interorgan Crosstalk: Roles in Health and Disease. J Am Med Dir Assoc 2016;17:789-796.

2. Cruz-Jentoft AJ, Baeyens JP, Bauer JM, Boirie $Y$, Cederholm T, Landi F, Martin FC, Michel JP, Rolland Y, Schneider SM, Topinková E, Vandewoude M, Zamboni M; European Working Group on Sarcopenia in Older People. Sarcopenia: European consensus on definition and diagnosis: Report of the European Working Group on Sarcopenia in Older People. Age Ageing 2010;39:412-423.

3. Cruz-Jentoft AJ, Bahat G, Bauer J, Boirie $Y$, Bruyère 0, Cederholm T, Cooper C, Landi F, Rolland Y, Sayer AA, Schneider SM, Sieber CC, Topinkova E, Vandewoude M, Visser M, Zamboni M; Writing Group for the European Working Group on Sarcopenia in Older People 2 (EWGSOP2), and the Extended Group for EWGSOP2. Sarcopenia: revised European consensus on definition and diagnosis. Age Ageing 2019;48:16-31.

4. Beaudart C, Zaaria M, Pasleau F, Reginster JY, Bruyère 0. Health Outcomes of Sarcopenia: A Systematic Review and Meta-Analysis. PLoS One 2017;12:0169548.

5. Chaudhry SI, Wang Y, Gill TM, Krumholz HM. Geriatric conditions and subsequent mortality in older patients with heart failure. J Am Coll Cardiol 2010;55:309-316.

6. Anker SD, Morley JE, von Haehling S. Welcome to the ICD-10 code for sarcopenia. J Cachexia Sarcopenia Muscle 2016;7:512-514.

7. Sanchez-Rodriguez D, Marco E, Cruz-Jentoft AJ. Defining sarcopenia: some caveats and challenges. Curr Opin Clin Nutr Metab Care 2020;23:127-132.

8. Perkisas S, De Cock A, Verhoeven V, Vandewoude M. Physiological and architectural changes in the ageing muscle and their relation to strength and function in sarcopenia. European Geriatric Medicine 2016;7:201-206.

9. Jager-Wittenaar H, Kasiukiewicz A, Landi F, Marco E, Merello A, Piotrowicz K Sanchez E, Sanchez-Rodriguez D, Scafoglieri A, Cruz-Jentoft A, Vandewoude M. Application of ultrasound for muscle assessment in sarcopenia: towards standardized measurements. Eur Geriatr Med 2018;9:739-757.

10. Bastijns S, De Cock AM, Vandewoude M, Perkisas S. Usability and Pitfalls of Shear-Wave Elastography for Evaluation of Muscle Quality and Its Potential in Assessing Sarcopenia: A Review. Ultrasound Med Biol 2020;46:28912907.

11. Perkisas S, Bastijns S, Baudry S, Bauer J, Beaudart C, Beckwée D, CruzJentoft $A$, Gasowski J, Hobbelen $H$, Jager-Wittenaar $H$, Kasiukiewicz $A$, Landi $F$, Małek M, Marco E, Martone AM, de Miguel AM, Piotrowicz K, Sanchez E, Sanchez-Rodriguez D, Scafoglieri A, Vandewoude M, Verhoeven V, Wojszel ZB, De Cock AM. Application of ultrasound for muscle assessment in sarcopenia: 2020 SARCUS update. Eur Geriatr Med 2021;12:45-59.

12. Akazawa N, Harada K, Okawa N, Tamura K, Moriyama H. Muscle mass and intramuscular fat of the quadriceps are related to muscle strength in nonambulatory chronic stroke survivors: A cross-sectional study. PLoS One 2018;13:0201789. 
13. Wilson DV, Moorey H, Stringer H, Sahbudin I, Filer A, Lord JM, Sapey E. Bilateral Anterior Thigh Thickness: A New Diagnostic Tool for the Identification of Low Muscle Mass? J Am Med Dir Assoc 2019;20:12471253.
14. Perkisas $S$, Baudry S, Bauer J, Beckwée D, De Cock AM, Hobbelen $H$, JagerWittenaar $H$, Kasiukiewicz A, Landi F, Marco E, Merello A, Piotrowicz $K$, Sanchez E, Sanchez-Rodriguez D, Scafoglieri A, Cruz-Jentoft A, Vandewoude M. The SARCUS project: evidence-based muscle assessment through ultrasound. Eur Geriatr Med 2019;10:157-158. 